\title{
MedienPädagogik
}

Zeitschrift für Theorie und Praxis der Medienbildung

www.medienpaed.com

Themenheft Nr. 34:

Forschung und Open Educational Resources - Eine Momentaufnahme für Europa

Herausgegeben von Markus Deimann

\section{Der Flipped Classroom als Motor für Open Educational Resources?}

\section{Eine Analyse der deutschsprachigen Lehrpersonencommunity}

Josef Buchner und Elke Höfler

\begin{abstract}
Zusammenfassung
Open Educational Resources und Flipped Classroom sind zwei in der aktuellen Bildungsforschung diskutierte Konzepte, die verstärkt auch den Weg ins Klassenzimmer finden. In sozialen Netzwerken, wie bei der Twitter-Community der Bildungspunks, werden Ideen mehr oder weniger offen getauscht und geteilt. In einer quantitativen Untersuchung wurde untersucht, inwieweit sich Flipped Classroom Anwenderinnen und Anwender von Nicht-Anwenderinnen und-Anwendern hinsichtlich der Einstellung zu OER unterscheiden. Es zeigt sich, dass Flipped Classroom Nutzerinnen und Nutzer eher bereit sind, freie und offene Bildungsressourcen zu produzieren und dann auch zu teilen.
\end{abstract}

The Flipped Classroom approach as engine for OER? An analysis of the Germanspeaking teaching community

\begin{abstract}
Open Educational Resources and the Flipped Classroom approach are two concepts that are actually very present in the discussion about education and educational change. In social networks, like the Twitter community Bildungspunks, ideas and materials are shared and exchanged. In this research it is analysed, if Flipped Classroom practitioners differ from non-practitioners regarding their attitude to OER. The analysed data reveals that teachers, who use the Flipped Classroom approach, are more willing to create and share their resources with the teaching community.
\end{abstract}

\section{Einleitung}

Ausgangspunkt der folgenden Überlegungen ist die subjektive und empirisch nicht belegte Beobachtung der diskursiven Interaktion einzelner Akteurinnen und Akteure im Bildungsbereich, die sich über den Microblogging-Dienst Twitter vernetzen und sich unter Nutzung unterschiedlicher Hashtags, wie beispielsweise \#twitterlehrerzimmer, \#twlz, \#EduPnx oder \#BayernEdu, austauschen: In den virtuellen 
Gesprächen zeigt sich, dass Open Educational Resources (OER) nicht erst infolge der jüngsten Urheberrechtsnovellen in Deutschland und Österreich thematisch im aktuellen Schulbetrieb angekommen zu sein scheinen (wie auch die Beliebtheit offener Werkzeuge, wie beispielsweise des OER-Arbeitsblattgenerators Tutory ${ }^{1}$ und der Video-Anreicherungsplattform H5P2, zeigen), sie werden von den Stakeholdern im Bildungsbereich jedoch selten offen geteilt (siehe hierzu die Frequenz der Beiträge in den Beitragsparaden der Bildungspunks ${ }^{3}$, die sich ihrer Eigendefinition zufolge als offene Austausch- und Vernetzungsplattform im deutschsprachigen Bildungssystem verstehen).

Wiewohl Markus Deimann (2018) in einem Artikel mit dem Titel «OER-Forschung Warum es sie bisher nicht gab und wie sich das ändern kann» eine breite Forschungslücke zu diesem Thema aufzeigt, lassen sich doch einige Teilbereiche ausmachen, die, wie auch Sandra Schön (2018) in einer Replik auf Deimanns Beitrag auf ihrem Blog schreibt, aktiv beforscht worden sind und werden.

Der Hochschulbereich erscheint dabei, rein quantitativ betrachtet, stärker beforscht als der Schulbereich: Von 231 Treffern in der Forschungsdatenbank ERIC ${ }^{4}$, die sich unter dem Suchbegriff «Open Educational Resources» unter Einschränkung auf «seit 2014 erschienen» und «Peer Review Only» finden, entfallen 136 auf die Hochschule und 109 auf die Postsecondary Education. Lediglich 14 Treffer entfallen schliesslich auf die Elementary Secondary Education sowie 12 auf Secondary Education, also in den Bereich der Sekundarstufe. In der Datenbank von Fachportal Pädagogik ${ }^{5}$ ist eine Einschränkung der Suche nach Zielgruppe nicht möglich, die vorhandenen 157 Beiträge des gleichen Beobachtungszeitraums weisen, nach einem Blick auf die paratextuelle Ausgestaltung, tendenziell ebenfalls eine Schwerpunktsetzung in den Bereichen Erwachsenenbildung sowie Hochschullehre auf.

Auf inhaltlicher Ebene fokussieren zahlreiche Publikationen eher die Rahmenbedingungen von Open Educational Resources (OER), wie beispielsweise die Finanzierung von Lehr- und Unterrichtsmaterialien (vgl. Kimmons 2015), die Möglichkeiten und Grenzen der Qualitätssicherung (vgl. Kimmons 2015; Mardis und Ambavarapu 2017) oder die Situation des Schul- bzw. Lehrbuchs, wie Neumann (2016) oder Heimstädt und Dobusch (2017) sowie die Machbarkeitsstudie zum OER-Schulbuch von Schön et al. (2017) zeigen. Auch wird das Potential von OER für ein spezifisches Gebiet, exemplarisch genannt seien Südafrika bei Goodier (2017) oder das Subsahara-Afrika bei Hassler et al. (2018), hervorgehoben.

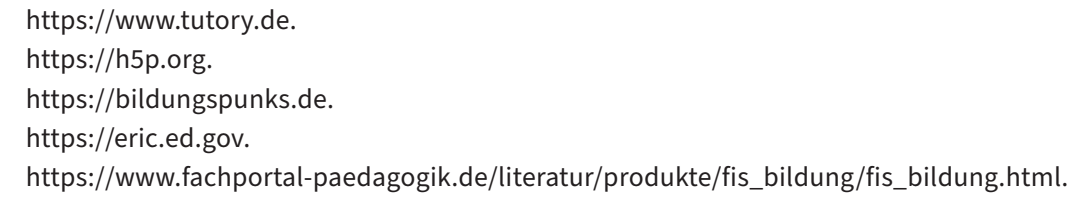


Eine Betrachtung des Themas OER aus (medien-)pädagogischer bzw. (medien-) didaktischer Perspektive fehlt jedoch weitgehend ebenso wie empirische Befunde zur konkreten Nutzung und/oder Erstellung von OER in didaktisierten Settings, wie auch von Deimann (2018) durch die Auswahl seiner Bibliographie implizit gezeigt wird. Einen ersten Schritt in Richtung Nutzungsanalyse hat die im September 2015 von Ebner et al. veröffentlichte Ist-Analyse zu freien Bildungsmaterialien (OER). Die Situation von freien Bildungsmaterialien (OER) in Deutschland in den Bildungsbereichen Schule, Hochschule, berufliche Bildung und Weiterbildung im Juni 2015 geleistet, doch stellt deren Aktualisierung mittlerweile ein latentes Forschungsdesiderat dar.

Ein didaktischer Zugang, in dessen Kontext OER untersucht wurden und der hier folglich eine Ausnahme darzustellen scheint, ist der Flipped Classroom. Beatriz de los Arcos (2014) hat vor einigen Jahren - und auf den amerikanischen Raum beschränkt - einen ersten Versuch unternommen, eine Verbindung von Flipped Classroom und OER herzustellen, indem sie Flipped Classroom-Akteurinnen und -Akteure nach der konkreten Nutzung von OER gefragt hat. Ihr Befund zeigt dabei, dass zwar beinahe die Hälfte der Befragten - und das Sample mit $n=109$ ist für den gesamten K-12-Bereich, also vom Kindergarten bis zur Hochschulreife, nicht besonders hoch - die für den Flipped Classroom selbst erstellten Materialien öffentlich teilt, jedoch nur knapp fünf Prozent als ausgewiesene OER.

Für den deutschsprachigen Raum fehlt eine derartige empirische Beschäftigung. Die von Helmut Zöhrer (2016) im Rahmen seiner Diplomarbeit mit dem Titel «Einsatz von Open Educational Resources mittels Flipped Classroom im Mathematikunterricht» durchgeführte Untersuchung fokussiert die Sicht der Schülerinnen und Schüler. Zöhrer beschreibt eine im konkreten schulischen Setting durchgeführte Untersuchung an einer Sekundarstufe in Graz und kommt dabei zum doppelten Schluss, dass a) Schülerinnen und Schüler das Gefühl haben, durch den Flipped Classroom weniger zu lernen als im Frontalunterricht und es b) keine Rolle spielt, ob die im Flipped Classroom angewandten Materialien als OER zur Verfügung gestellt werden oder nicht. Zöhrer $(2016,83)$ resümiert:

"Obwohl zu Beginn der Studie auf die Sinnhaftigkeit von OER und dass es sich sowohl bei den Lernvideos als auch bei den digitalen Skripten um eben solche handelt hingewiesen wurde, wirkten die Lernenden davon wenig beeindruckt. So wurde auch im weiteren Verlauf der Studie kaum über OER gesprochen. Natürlich hätten die Schüler/innen - gleich wie beliebige Interessierte weltweit - die Möglichkeit, die Materialien für eigene Zwecke zu verwenden und abzuändern; allerdings ist nicht bekannt, ob diese Chance tatsächlich bereits in Anspruch genommen wurde.»

Trotz aller Einschränkungen, die dieser Diplomarbeit zugrunde liegen, ist sie ein interessanter Befund aus Perspektive der Lernenden, die Lehrpersonensicht wird jedoch nicht reflektiert. 
Eben diese Perspektive soll mit dem vorliegenden Beitrag eingenommen werden. Dabei leiten zwei Forschungshypothesen, die die Ergebnisse von de los Arcos (2014) direkt aufnehmen, das Erkenntnisinteresse des Autors und der Autorin und bilden den Ausgangspunkt einer quantitativen Erhebung im deutschsprachigen Raum:

1. Lehrende, die das Modell des Flipped Classroom (FC) praktizieren, unterscheiden sich von Nicht-Praktizierenden in den Bereichen:

a. Selbstwirksamkeit (bezogen auf Wissen und Fähigkeiten zu OER)

b. Bereitschaft zu teilen

c. Offenheit (bezogen auf die Auswahl der Lizenz)

2. Lehrende, die Materialien für den FC selbst erstellen, unterscheiden sich in den drei oben genannten Aspekten von jenen FC-Anwendenden, die ihre Materialien nicht selber erstellen.

Zur Verifizierung oder Falsifizierung der beiden Hypothesen wird in den folgenden beiden Abschnitten zum einen die Rolle von OER in der Schule - unter Berücksichtigung von Openness als Konzept - betrachtet, bevor zum anderen ein Forschungsabriss zu Flipped Classroom und Schule gegeben wird. Der Schwerpunkt liegt dabei auf der Sekundarstufe, wenngleich der Blick über den Tellerrand, vor allem in die Lehramtsausbildung, notwendigerweise nicht ausbleibt. Im vierten Abschnitt wird daraufhin das Forschungsdesign der durchgeführten quantitativen Erhebung zum Thema OER und Flipped Classroom beschrieben und die Ergebnisse werden diskutiert. Den Abschluss dieses Beitrags bilden neben der Interpretation der Daten und Besprechung der Forschungshypothesen vor allem die Formulierung der Limitationen der Arbeit sowie das Aufzeigen weiterführender Forschungsdesiderate.

\section{OER in der schulischen Praxis}

Bevor der Blick auf den Flipped Classroom als methodischen Ansatz fällt, soll einführend der schulischen Praxis in der Erstellung von Open Educational Resources nachgegangen werden. Ausgangspunkt der Überlegungen ist die von Sandra Peter und Markus Deimann (2013) formulierte Aussage, wonach Openness im Bildungssystem bereits zur Selbstverständlichkeit geworden sei:

«So why reflect on a topic [gemeint: Openness, Anm. d. Verf.] that seems so commonly used in education nowadays and obviously no educator would earnestly reject the importance of openness for education? Should we not be concerned with issues that are beyond openness such as new educational practices? As illustrated by the current debate on «Flipped Classroom` [...], openness (here in the form of open access and OER), is more or less taken for granted and questions like (What is the best use of our class time? are now on top of the research agenda.» 
Betrachtet man hierzu zum einen die vorhandenen Forschungsergebnisse und zum anderen jene Kanäle, über die einzelne Praktikerinnen und Praktiker aus dem schulischen Alltag berichten, ihre (Edu-)Blogs, so zeigt sich, dass der Aussage zu widersprechen ist: Openness, in Form von beispielsweise Open Access und auch OER, ist noch keine Selbstverständlichkeit in (schulischen) Unterrichtssettings. Dies mag auch an der Schwierigkeit liegen, open oder offen als Attribut zu definieren. Longhino (2018, 6) zeigt dies für den Bereich offener Lernformen, indem sie in Anlehnung an Peschel (2012) unterschiedliche Dimensionen von Offenheit nennt, nämlich Offenheit der Organisation, der Methode, des Inhalts, des sozialen Gefüges und der Persönlichkeit. Beatriz de los Arcos et al. $(2016,38)$ weisen in ihrer Untersuchung dezidiert auf ein fehlendes Bewusstsein für und die fehlende Kenntnis um die Dimensionen von OER hin:

«Actually, although the question was put to only a subgroup of survey participants ( $n=193)$, over half of them reported that either they had never seen a CC logo or that they had seen it but did not know what it meant.»

Sie schlussfolgern, dass nicht nur ein Bewusstsein für Offenheit und OER geschaffen werden muss, sondern auch die Mentalität der Lehrenden einer Veränderung bedarf. "Consequently, whether teachers are unintentionally or deliberately shunning open licenses, this disparity between knowing about open practices and engaging in open practices bolsters the argument that mainstreaming OER is not only a matter of raising awareness of openness but of changing teachers' habits.»

Die Lehrpersonen adaptieren Materialien, die sie im Internet finden, was unter Berücksichtigung des amerikanischen Copyrights im Sinne der Fair Use-Regelung in bestimmten Kontexten auch möglich ist, um sie an die Bedürfnisse ihrer Lernenden anzupassen, das Bewusstsein für «starke OER» (Kerres 2016), also Open Educational Resources, die die von David Wiley (2014) als 5R formulierten Kriterien für OER, nämlich Retain, Reuse, Revise, Remix, Redistribute, berücksichtigen und erlauben, und der Zugang zur offenen Lizenzierung eigener Materialien fehlen dabei jedoch. Ähnliches halten Jöran Muuß-Merholz und Sandra Schön $(2015,39)$ fest:

«Während die Debatte um kostenlose und frei lizenzierte Bildungsmaterialien in anderen Bildungsbereichen relativ differenziert erfolgt, wird im Schulbereich der Begriff OER häufig für alle Materialien verwendet, die kostenlos im Internet zu finden [Hervorhebung im Original] sind.»

Der Autor und die Autorin identifizieren dabei vier Fallstricke in Hinblick auf die Verbreitung von OER in der Schule: die Qualitätssicherung, Lizenzierung und Rechtssicherheit, Qualifizierungsmodelle sowie Geschäftsmodelle und folgen damit den Ergebnissen von Van Acker et al. (2013) sowie (Weller et al. 2015). 
Marc Hankmann, Redaktionsleiter von MediaLABCom und ehemaliger Chefredakteur des B2B-Informationsdienstes Digital Insider, betitelt einen 2014 auf Bildung plus erschienenen Artikel mit «Frei, kostenlos - aber auf Halde. Potenzial von OER und warum sie so selten für den Unterricht genutzt werden» und kommt zum Schluss:

«Da die Verlage nicht hinter OER stehen, gibt es auch nur wenig Beachtung seitens der Politik, sprich: Es fehlt die finanzielle Förderung durch die öffentliche Hand. So lange ist OER auf motivierte Lehrer angewiesen, die ihr eigenes Material erstellen und online verbreiten.»

Dass der finanzielle Aspekt nicht primär ausschlaggebend ist, wenn es um das Teilen von selbst erstellten Materialien geht, zeigen Frederik Van Acker et al. (2013) in ihrer Untersuchung mit dem Titel «Why teachers share educational resources: A social exchange perspective». Ausgehend von der Annahme, dass die drei Faktoren Costs, Rewards und Trust das Teilverhalten beeinflussen, kommen die Autorinnen und Autoren zu einem überraschenden Schluss: «It seems that altruistic motives play a key role in teachers' intentions to share OER. This implies that teachers enjoy the behaviour as such, without the need for any extrinsic incentives.» $(2013,188)$ Es sei, so die Autorinnen und Autoren, wichtig, aufzuzeigen, dass Kolleginnen und Kollegen ihre Inhalte teilen, um die intrinsische Motivation der anderen, die eigenen Inhalte ebenfalls zu teilen, zu erhöhen. Die Kosten spielen hingegen «only a limited role» (2013, 188). Die Autorinnen und Autoren schlagen als Fazit vor, die Lehrenden in Hinblick auf die Erstellung von Materialien zu schulen und hierbei zu unterstützen, um qualitativ hochwertiges Material zu erstellen, das geteilt und von anderen auch verwendet wird, was in Hinblick auf die Wertschätzung und folglich die intrinsische Motivation eine zentrale Rolle spiele. Letztlich scheitert es in der Praxis, wie der Schweizer Lehrer und Mediendidaktiker Philippe Wampfler (2015) zeigt, auch am grundsätzlichen Willen, Materialien zu erstellen und zu teilen - unabhängig ob OER oder nicht. Als Gründe nennt Wampfler u.a. rechtliche Unsicherheiten, das Gefühl der Unzulänglichkeit der eigenen Materialien, die Angst vor dem kritischen Blick einer fremden Person.

Die Rolle der Schulung nennt auch Dominik Neumann (2016), der eine Marktanalyse durchgeführt hat, in deren Zuge er sich mit der Frage beschäftigt hat, wer für welche Bereiche in welcher Form Materialien als OER zur Verfügung stellt. Dabei zeigt er, dass der Bedarf an OER in der Schule anscheinend einer fachspezifischen Schwankung unterliegt, da vor allem die Sprachfächer einen besonderen Zuwachs erlebt haben, wenngleich der Autor einschränkt, dass in dieser Analyse auf das Verhältnis von Angebot und Nachfrage zu achten sei. 
Beatriz de los Arcos et al. (2016, 37f.) erkennen für ihre Zielgruppe einen weiteren Unterschied, wenn sie zwischen Lehrenden in Online- und Präsenz-Unterrichtssettings unterscheiden:

"The discovery that face-to-face teachers create and adapt OER less than their online and blended counterparts may well substantiate the relation between adapting OER and personalized learning. Assuming that K-12 face-to-face classrooms are often locked into using textbooks from a particular publisher, perhaps the need for face-to-face teachers to produce their own resources is less immediate.»

Wenngleich an dieser Stelle die Argumentation stark auf das amerikanische Bildungssystem fokussiert, zeigt sich auf einer abstrahierenden Ebene, dass Lehrpersonen, die, im weitesten Sinne des Verständnisses, eLearning betreiben, stärker OER produzieren als Lehrpersonen, die primär Face-to-Face unterrichten. Der Flipped Classroom als ein, den virtuellen und realen Lehr- und Lernraum verbindender methodischer Ansatz würde also, so die verkürzte Ableitung, tendenziell für die Erstellung von OER sprechen.

\section{Flipped Classroom-Akteurinnen und -Akteure und ihr Beitrag zu OER}

Der methodisch-didaktische Ansatz, den traditionellen Unterricht auf den Kopf zu stellen, stammt ursprünglich aus dem amerikanischen Hochschulbereich. Lage, Platt und Treglia (2000) stellten im Rahmen einer Lehrveranstaltung an der Universität Miami ihren Studierenden Videos als Begleitmaterial zu Lehrbuchpassagen zur Verfügung. Sie bezeichneten ihre Idee als Inverting the Classroom, da die Videos zur Vorbereitung auf die Präsenztermine verwendet wurden. Dieses Konzept wurde von Bergmann und Sams (2012) für den Schulunterricht adaptiert und Flipped Classroom bezeichnet. Angelehnt an diese frühe begriffliche Differenzierung zwischen Schule und Hochschule wird im deutschen Sprachraum für den Bereich der Hochschullehre meist der Begriff Inverted Classroom (vgl. Handke 2014), für den schulischen Bereich meist Flipped Classroom verwendet. Um etwaige Begriffsverwirrungen zu vermeiden, wird in diesem Beitrag durchgängig der Begriff Flipped Classroom (FC) verwendet, auch wenn Ergebnisse aus dem Bereich der Hochschule vorgestellt werden.

Für die Umsetzung des umgedrehten Unterrichts, wie diese Unterrichtsform im Deutschen auch genannt wird, greifen die meisten Anwenderinnen und Anwender auf Videos zurück, die sie z.B. auf Videoplattformen wie YouTube finden oder selbst produzieren (vgl. O'Flaherty und Phillips 2015; de los Arcos 2014). Diese werden den Lernenden vor dem eigentlichen Unterricht zur Verfügung gestellt, um die gemeinsame Zeit in der Klasse oder im Seminar für aktivierende Lehr- und Lernmethoden zu nutzen (vgl. van Treeck, Himpsl-Gutermann, und Robes 2013; Fischer und Spannagel 2012). 
Bei diesem methodisch-didaktischen Ansatz entstehen sowohl für die Lernenden als auch für die Lehrenden einige Herausforderungen. Lernende kommen oft unvorbereitet in die Präsenzphase und fühlen sich aufgrund der fehlenden Möglichkeit, Fragen zu stellen, alleine gelassen. Für Lehrpersonen ist der erstmalige Einsatz des Flipped Classroom die grösste Hürde, da die Gestaltung der Vorbereitungsphase mithilfe digitaler Medien einen zeitlichen Mehraufwand mit sich bringt, insbesondere wenn die zu verwendenden Materialien selbst produziert werden müssen (vgl. Lo, Hew, und Chen 2017). Bei de los Arcos (2014) gaben von 109 amerikanischen Lehrpersonen des Flipped Learning Network (FLN) 83,3\% (75) an, dass sie die Videos für die Umsetzung ihres Flipped Classroom selbst produzieren. Weiters wurden Stundenbilder, Quizze, Spiele und Bilder gestaltet, jedoch weitaus weniger oft als Videos. $43,3 \%$ (42) der Lehrkräfte gaben an, die Materialien auch online zu publizieren und zu teilen. Der Anteil jener, die dies auch unter einer Creative-Commons-Lizenz tun, fällt mit 5,1\% (5) gering aus. Dieses Ergebnis ist deshalb so interessant, da 82,5\% (90) der Befragten der gleichen Stichprobe angaben, Online-Ressourcen nur nach einer entsprechenden Anpassung zu nutzen. Diese Anpassung bzw. Erlaubnis der Bearbeitung ist u.a. nach Geser (2007) und Wiley (2014) eines der wichtigsten Kennzeichen für OER und scheint entsprechend den Ergebnissen dieser Studie auch für Lehrende ein wichtiger Faktor für die Nutzung bereits vorhandener Materialien zu sein.

Wie bereits skizziert, sind Videos das am häufigsten genutzte Medium für die Vorbereitungsphase im umgedrehten Unterricht. Daher werden im Folgenden auszugsweise YouTube-Kanäle der deutschsprachigen Flipped-Classroom-Praktizierenden vorgestellt, stets mit Angabe der Lizenzierung. Buchner und Freisleben-Teutscher (2017) haben mit einem Beitrag bereits Vorarbeit geleistet und einige Kanäle analysiert. Diese Analyse wird nun erneut aufgegriffen und um weitere Akteurinnen und Akteure ergänzt. Die Auswahl der Video-Channels für den schulischen Bereich erfolgt auf der Grundlage der Vernetzungsseiten http://www.flipped-classroom-austria. at und https://www.umgedrehterunterricht.de, für den Hochschulbereich werden Beitragende der internationalen Konferenz «Inverted Classroom \& Beyond» berücksichtigt. Insgesamt wurden 16 schulische und sechs hochschulische YouTube-Kanäle betrachtet, für eine Übersicht siehe Tabelle 1.

\begin{tabular}{|l|l|l|l|}
\hline Institution & Fach/Fächer & Lizenz & Link \\
\hline Schule & Mathematik & CC & $\begin{array}{l}\text { https://www.youtube.com/user/ } \\
\text { DaHugo82 }\end{array}$ \\
\hline Schule & Geschichte & CC & $\begin{array}{l}\text { https://www.youtube.com/channel/ } \\
\text { UCX-rN8GX2TfSvkvxrt3ytwQ }\end{array}$ \\
\hline Schule & Angewandte Informatik & $\begin{array}{l}\text { Standard YouTube } \\
\text { Lizenz }\end{array}$ & $\begin{array}{l}\text { https://www.youtube.com/channel/ } \\
\text { UCdwK7qpRK96VEB98DupBKXA/ }\end{array}$ \\
\hline Schule & Mathematik & $\begin{array}{l}\text { Standard YouTube } \\
\text { Lizenz }\end{array}$ & $\begin{array}{l}\text { https://www.youtube.com/channel/ } \\
\text { UCoCj6f4kmzTa691LZ3Lgtzg/ }\end{array}$ \\
\hline
\end{tabular}




\begin{tabular}{|c|c|c|c|}
\hline Schule & Mathematik & $\mathrm{CC}$ & $\begin{array}{l}\text { https://www.youtube.com/user/ } \\
\text { kurtsoeser }\end{array}$ \\
\hline Schule & $\begin{array}{l}\text { Mathematik, Geogra- } \\
\text { phie, Deutsch, Englisch }\end{array}$ & $\begin{array}{l}\text { Standard YouTube } \\
\text { Lizenz }\end{array}$ & $\begin{array}{l}\text { https://www.youtube.com/channel/ } \\
\text { UC4FmX2u_86VYSi2pZidUa2g }\end{array}$ \\
\hline Schule & Englisch & $\begin{array}{l}\text { Standard YouTube } \\
\text { Lizenz }\end{array}$ & $\begin{array}{l}\text { https://www.youtube.com/channel/ } \\
\text { UCNWDHbFId90XfkXSgP_S_DA/ }\end{array}$ \\
\hline Schule & Mathematik & $\begin{array}{l}\text { Standard YouTube } \\
\text { Lizenz }\end{array}$ & $\begin{array}{l}\text { https://www.youtube.com/channel/ } \\
\text { UCzurVOarwR28nXDYsRWTTjw }\end{array}$ \\
\hline Schule & Mathematik & $\begin{array}{l}\text { Standard YouTube } \\
\text { Lizenz }\end{array}$ & $\begin{array}{l}\text { https://www.youtube.com/channel/ } \\
\text { UCge5loR9wYKLHfK4erOennQ }\end{array}$ \\
\hline Schule & $\begin{array}{l}\text { Deutsch und Textverar- } \\
\text { beitung }\end{array}$ & CC & $\begin{array}{l}\text { https://www.youtube.com/user/ } \\
\text { flipclass }\end{array}$ \\
\hline Schule & BwR & $\begin{array}{l}\text { CC\& Standard YouTu- } \\
\text { be Lizenz }\end{array}$ & $\begin{array}{l}\text { https://www.youtube.com/user/ } \\
\text { ryceps }\end{array}$ \\
\hline Schule & Deutsch und Geschichte & $\begin{array}{l}\text { Standard YouTube } \\
\text { Lizenz }\end{array}$ & $\begin{array}{l}\text { https://www.youtube.com/channel/ } \\
\text { UCE7jjn8-XVBWrOdSkBLz-Mw }\end{array}$ \\
\hline Schule & Physik, Deutsch & $\begin{array}{l}\text { Standard YouTube } \\
\text { Lizenz }\end{array}$ & $\begin{array}{l}\text { https://www.youtube.com/channel/ } \\
\text { UC8c41GXQS7P0DGu3cJcVIPg }\end{array}$ \\
\hline Schule & BwR & $\begin{array}{l}\text { Standard YouTube } \\
\text { Lizenz }\end{array}$ & $\begin{array}{l}\text { https://www.youtube.com/channel/ } \\
\text { UCiFPshRgLk63jgbwT52yGUQ }\end{array}$ \\
\hline Schule & Mathematik & $\begin{array}{l}\text { Standard YouTube } \\
\text { Lizenz }\end{array}$ & $\begin{array}{l}\text { https://www.youtube.com/channel/ } \\
\text { UC5yt3JsEl-84e9gN0J0iTEA }\end{array}$ \\
\hline Schule & Mathematik & $\begin{array}{l}\text { Standard YouTube } \\
\text { Lizenz }\end{array}$ & $\begin{array}{l}\text { https://www.youtube.com/channel/ } \\
\text { UCw-2nqxX97yy1fXRYzajJqw/ }\end{array}$ \\
\hline Hochschule & Linguistik & CC & $\begin{array}{l}\text { https://www.youtube.com/user/ } \\
\text { LinguisticsMarburg }\end{array}$ \\
\hline Hochschule & Ingenieursmathematik & $\begin{array}{l}\text { Standard YouTube } \\
\text { Lizenz }\end{array}$ & $\begin{array}{l}\text { https://www.youtube.com/user/ } \\
\text { JoernLoviscach }\end{array}$ \\
\hline Hochschule & Mathematik & $\mathrm{CC}$ & $\begin{array}{l}\text { https://www.youtube.com/user/ } \\
\text { pharithmetik }\end{array}$ \\
\hline Hochschule & $\begin{array}{l}\text { Diätologie, Physiothera- } \\
\text { pie, Medientechnik, etc. }\end{array}$ & $\mathrm{CC}$ & $\begin{array}{l}\text { https://www.youtube.com/user/ } \\
\text { FHStPoelten }\end{array}$ \\
\hline Hochschule & Statistik & $\begin{array}{l}\text { Standard YouTube } \\
\text { Lizenz }\end{array}$ & $\begin{array}{l}\text { https://www.youtube.com/user/ } \\
\text { Methodenlehre }\end{array}$ \\
\hline Hochschule & Statistik & $\begin{array}{l}\text { Standard YouTube } \\
\text { Lizenz }\end{array}$ & $\begin{array}{l}\text { https://www.youtube.com/channel/ } \\
\text { UCBF5kyHKIepC4qLT-C2Am_w/ }\end{array}$ \\
\hline
\end{tabular}

Tab. 1.: Übersicht über die FC-Kanäle (Stand von Mai 2018).

An dieser Stelle muss festgehalten werden, dass die folgende Analyse keinen Anspruch auf Vollständigkeit erhebt. Sehr wohl können aber Tendenzen aus Tabelle 1 abgeleitet werden. Ein Kanal aus dem schulischen Bereich verwendet sowohl eine Creative-Commons-Lizenz, als auch die standard YouTube-Lizenz. Dieser Kanal wird daher für die prozentuelle Auswertung nicht berücksichtigt. Von den übrigen 
15 praktizierenden Flipped-Classroom-Lehrpersonen mit eigenem YouTube-Kanal kennzeichnen vier ihre Videos mit einer CC-Lizenz, dies entspricht 26,66\%. Für die Hochschullehre wurden sechs YouTube-Kanäle gesammelt, drei weisen die vorhandenen Videos als CC-Materialien aus, dies entspricht $50 \%$.

All diese Lehrpersonen verbindet, dass sie selbst Videos für ihre Lernenden produzieren und diese dann auch öffentlich im Netz für andere zur Verfügung stellen. Durch die Bereitstellung auf einem eigenen YouTube-Kanal erfüllen sie ein von Wiley (2014) genanntes Kriterium für OER, nämlich den freien Zugang zu Wissen zu unterstützen. Inwieweit das Kriterium der Offenheit allgemein auf die Flipped-ClassroomCommunity, d.h. jene, die diesen methodisch-didaktischen Zugang in ihrem Unterricht bzw. der Lehre einsetzen, zutrifft, verlangt nach einer weiteren Untersuchung. Tabelle 1 zeigt weiters, unter Berücksichtigung des niedrigen Samples, dass von Hochschullehrenden produzierte Materialien neben dem freien Zugang auch das Kriterium der Offenheit öfter erfüllen.

\section{Studie}

\section{Stichprobe und methodisches Vorgehen}

Für die Erhebung der Daten wurde ein Online-Fragebogen mit dem Tool LimeSurvey gestaltet. 361 vollständig ausgefüllte Datensätze wurden für die Analyse berücksichtigt, das Durchschnittsalter der Teilnehmenden lag zum Zeitpunkt der Erhebung im März und April 2018 bei 47,2 Jahren (SD = 9,99, Minimum 22 Jahre, Maximum 70 Jahre). Der Fragebogen wurde mittels Short-Link über Social-Media-Kanäle (Facebook und Twitter), sowie über die Mailing-Listen der Virtuellen Pädagogischen Hochschu$l^{6}{ }^{6}$ und der Initiative eEducation ${ }^{7}$ Austria des österreichischen Bundesministeriums für Bildung, Wissenschaft und Forschung zugänglich gemacht. Weiters haben die Autorin und der Autor über Kolleginnen und Kollegen aus Deutschland, der Schweiz und Luxemburg auch für Schulen dieser Länder den Link zur Verfügung gestellt. Mit 257 beantworteten Fragebögen machen in Österreich unterrichtende Lehrpersonen den Grossteil der Stichprobe aus. Aus Deutschland kamen 82 Antworten, aus der Schweiz 19, aus Luxemburg zwei und ein Fragebogen wurde von einer Lehrperson beantwortet, die in Italien unterrichtet. Um ein detaillierteres Bild über die Verwendung und den Einsatz von Flipped Classroom und OER zu bekommen, wurden die Teilnehmenden gefragt, in welcher Schulstufe sie unterrichten. Hier waren auch Mehrfachnennungen möglich, da in der Praxis immer wieder kombinierte Anstellungsverhältnisse zu finden sind. Die deskriptive Statistik wurde mit SPSS 24 gerechnet.

6 http://www.virtuelle-ph.at.

7 https://eeducation.at. 


\section{Ergebnisse}

Im Fragebogen wurden die Teilnehmenden zunächst nach ihrem theoretischen Wissen zu OER und Flipped Classroom befragt, ehe dann die praktische Anwendung die Stichprobe in zwei Gruppen aufteilte. Zum einen in Flipped-Classroom-Anwendende, zum anderen jene, die den umgedrehten Unterricht bis zum Zeitpunkt der Erhebung noch nicht in ihrer Unterrichtspraxis eingesetzt hatten. Tabelle 2 gibt einen Überblick über die Verteilung nach Bildungseinrichtung, das jeweilige Durchschnittsalter und den Anteil an Flipped-Classroom-Anwendende.

\begin{tabular}{|l|l|l|l|l|}
\hline $\begin{array}{l}\text { Bildungsbe- } \\
\text { reich }\end{array}$ & Anzahl & $\begin{array}{l}\text { Davon weib- } \\
\text { lich }\end{array}$ & $\begin{array}{l}\text { Durchschnitts- } \\
\text { alter in Jahren } \\
\text { (SD) }\end{array}$ & $\begin{array}{l}\text { FC-Anwende- } \\
\text { rinnen und } \\
\text {-Anwender }\end{array}$ \\
\hline Primarstufe & 74 & 54 & $48,74(9,17)$ & $18(24,32 \%)$ \\
\hline Sekundarstufe 1 & 209 & 109 & $46,80(10,52)$ & $108(51,67 \%)$ \\
\hline Sekundarstufe 2 & 123 & 53 & $44,34(9,96)$ & $79(64,23 \%)$ \\
\hline Tertiäre Bildung & 82 & 30 & $45,38(9,74)$ & $62(75,61 \%)$ \\
\hline
\end{tabular}

Tab. 2.: Bildungsbereich, Durchschnittsalter und FC-Anwendende. ${ }^{8}$

Wie Tabelle 2 zeigt, wird der umgedrehte Unterricht innerhalb der Stichprobe in der Sekundarstufe 1 und 2 deutlich häufiger verwendet als in der Primarstufe. Auch ersichtlich ist, dass der Anteil der FC-Anwendenden im Tertiären Bildungsbereich am höchsten ist. Laut dieser Befragung setzen ca. 75\% der Hochschullehrpersonen diese Methode ein, knapp gefolgt von Lehrpersonen der Sekundarstufe 2 (ca. 64\%). In der Sekundarstufe 1 nutzt jede und jeder Zweite den FC praktisch, in der Primarstufe gut ein Viertel der befragten Lehrpersonen.

Insgesamt geben 191 Personen an, bereits FC in der Praxis einzusetzen, demgegenüber stehen 170 Nicht-Nutzende. Tabelle 3 zeigt die verwendeten Items der Skalen Selbstwirksamkeit und Bereitschaft zu teilen, Cronbachs Alpha für die Skalen sowie die Mittelwerte mit Standardabweichung. Verwendet wurden LIKERT-Skalen von 1 (Stimme ganz zu) bis 5 (Stimme gar nicht zu), niedrige Werte stehen demnach für Zustimmung.

8 Bei der Darstellung in dieser Tabelle werden Kombinationen noch nicht berücksichtigt, daher übersteigt die Summe in der Spalte Anzahl auch die Gesamtstichprobenanzahl von 361. 


\begin{tabular}{|l|l|l|l|}
\hline Skala (Items) & $\begin{array}{l}\text { Cronbachs } \\
\text { Alpha }\end{array}$ & $\begin{array}{l}\text { FC (n= } \\
\text { 191) }\end{array}$ & $\begin{array}{l}\text { Nicht-FC } \\
\text { (n= 170) }\end{array}$ \\
\hline $\begin{array}{l}\text { Selbstwirksamkeit } \\
\text { Open Educational Resources (OER) sind mir } \\
\text { theoretisch vertraut. } \\
\text { Meine produzierten Materialien können andere } \\
\text { Lehrkräfte in der Vorbereitung und Durchfüh- } \\
\text { rung ihres Unterrichts unterstützen. } \\
\begin{array}{l}\text { Ich habe die notwendigen Fähigkeiten und Fer- } \\
\text { tigkeiten, um digitale Lehr- und Lernmaterialien } \\
\text { zu erstellen. } \\
\text { Ich habe die notwendigen Fähigkeiten und Fer- } \\
\text { tigkeiten, um digitale Lehr- und Lernmaterialien } \\
\text { online zu teilen. }\end{array}\end{array}$ & 0.8 & $1.9(0.9)$ & $2.7(1.0)$ \\
\hline $\begin{array}{l}\text { Bereitschaft zu teilen } \\
\text { Wenn ich (digitale) Lehr- und Lernmaterialien } \\
\text { produziere, gebe ich diese frei zugänglich weiter. } \\
\text { Wenn ich vorhandene (digitale) Materialien } \\
\text { verändere, stelle ich sie im Anschluss wieder für } \\
\text { andere frei zugänglich zur Verfügung. }\end{array}$ & 0.8 & $2.5(1.2)$ & 2.8 (1.4) \\
\hline
\end{tabular}

Tab. 3.: Gegenüberstellung der FC- und Nicht-FC-Gruppe hinsichtlich Selbstwirksamkeit und Bereitschaft zu teilen.

Um die Offenheit zu erheben, wurden die Teilnehmenden gebeten, eine Lizenz zu wählen, die sie selbst erstellten Materialien zuweisen würden. Die Anzahl der Antworten unterscheidet sich für dieses Item von den anderen beiden, da nur jene Fragebögen berücksichtigt wurden, in denen auch eine Zuteilung gemacht wurde. Keine Zuteilung war möglich, wenn die Personen entweder noch nichts über die verschiedenen Lizenzen wussten oder sie keine Antwort abgeben wollten, weil diese Entscheidung von vielen Faktoren abhängig ist und von Fall zu Fall entschieden wird (laut Bezeichnung dieser Items). Tabelle 4 zeigt die Ergebnisse für beide Gruppen mit der Anzahl der gewählten Lizenzen und den Mittelwerten mit Standardabweichung.

\begin{tabular}{|l|l|l|}
\hline & FC $(\mathbf{n}=118)$ & Nicht-FC $(\mathbf{n}=\mathbf{5 8})$ \\
\hline Offenheit - welche Lizenz wird vergeben? & $1.4(0.8)$ & $1.8(0.9)$ \\
CCO & $5 \%$ & $3 \%$ \\
CC BY-SA & $58 \%$ & $45 \%$ \\
CC BY-ND & $25 \%$ & $22 \%$ \\
C & $12 \%$ & $30 \%$ \\
\hline
\end{tabular}

Tab. 4.: Gegenüberstellung der FC- und Nicht-FC-Gruppe hinsichtlich Offenheit.

Um Hypothese 1 zu testen, werden die Mittelwerte der beiden Gruppen für jede Skala miteinander verglichen. FC-Anwendende unterscheiden sich signifikant von NichtFC-Anwendenden bei der berichteten Selbstwirksamkeit (Mann-Whitney-U-Test: U = 
8781.500, $p=0.000$ ). Keine signifikanten Unterschiede konnten für die Skala Bereitschaft zu teilen gefunden werden (Mann-Whitney-U-Test: $U=14495.500, p=0.075$ ). Signifikant sind die Unterschiede bezüglich der gewählten Lizenzierungen (MannWhitney-U-Test: $U=2769.000, p=0.024$ ).

Für die Testung der zweiten Hypothese werden nun jene FC-Anwendenden betrachtet, die für ihre Umsetzung eigene Materialien produzieren. Von den 191 FCAnwendenden haben 154 angegeben, entweder selbst Materialien zu produzieren oder nicht. Die restlichen 37 haben hier die Option «weder noch» gewählt. Die neue Stichprobe teilt sich in 120 produzierende Flipped-Classroom-Anwendende und 34 Nicht-Produzierende für die Skalen Selbstwirksamkeit und Bereitschaft zu teilen. Die Ergebnisse sind in Tabelle 5 dargestellt.

\begin{tabular}{|l|l|l|}
\hline Skala (Items) & $\begin{array}{l}\text { FC mit Produkti- } \\
\text { on }(\mathbf{n}=120)\end{array}$ & $\begin{array}{l}\text { FC ohne Pro- } \\
\text { duktion }(\mathbf{n}=\mathbf{3 4})\end{array}$ \\
\hline Selbstwirksamkeit & $1.6(0.6)$ & $2.8(1.1)$ \\
\hline Bereitschaft zu teilen & $2.4(1.1)$ & $3.1(1.4)$ \\
\hline
\end{tabular}

Tab. 5.: Gegenüberstellung der Gruppen FC mit Produktion und FC ohne Produktion hinsichtlich Selbstwirksamkeit und Bereitschaft zu teilen.

Bei der Darstellung der Ergebnisse zur Offenheit wurde wieder auf jene Fragebögen verzichtet, die keine Angabe zur Lizenz machten. Die verbleibende Anzahl an Antworten sowie die Mittelwerte mit Standardabweichung und der prozentuellen Verteilung der Lizenzen sind in Tabelle 6 ersichtlich.

\begin{tabular}{|l|l|l|}
\hline & $\begin{array}{l}\text { FC mit Produkti- } \\
\text { on }(\mathbf{n}=84)\end{array}$ & $\begin{array}{l}\text { FC ohne Produk- } \\
\text { tion }(\mathbf{n}=17)\end{array}$ \\
\hline Offenheit - welche Lizenz wird vergeben? & $1.3(0.7)$ & $2.1(0.8)$ \\
CCO & $7 \%$ & $0 \%$ \\
CC BY-SA & $66 \%$ & $29 \%$ \\
CC BY-ND & $20 \%$ & $35 \%$ \\
( & $7 \%$ & $36 \%$ \\
\hline
\end{tabular}

Tab. 6.: Gegenüberstellung der Gruppen FC mit Produktion und FC ohne Produktion hinsichtlich Offenheit.

Um Hypothese 2 zu überprüfen, werden erneut die Mittelwerte der beiden Gruppen für jede Skala verglichen. Für die Skala Selbstwirksamkeit ist ein signifikanter Unterschied zwischen den beiden FC-Gruppen zu erkennen (Mann-Whitney-U-Test: $\mathrm{U}=$ $828.000, p=0.000$ ). Ebenso unterscheiden sich die beiden Gruppen signifikant hinsichtlich der Bereitschaft zu teilen (Mann-Whitney-U-Test: $U=1460.000, p=0.011$. Auch bei der Vergabe der Lizenzen unterscheiden sich die produzierenden FC-Anwendenden signifikant von den Nicht-Produzierenden (Mann-Whitney-U-Test: $U=$ $357.500, p=0.000$ ). 
Die Werte aller drei Skalen wurden als Kontrolle mit den Werten der Nicht-FCGruppe (Tabelle 3 und 4) verglichen. Für alle drei Skalen zeigten sich signifikante Unterschiede zwischen der Gruppe FC mit Produktion und der Gruppe Nicht-FC. Keine signifikanten Unterschiede wurden zwischen der Gruppe FC ohne Produktion und der Gruppe Nicht-FC gefunden.

\section{Diskussion der Ergebnisse}

In diesem Abschnitt wird diskutiert, ob die gefundenen Ergebnisse die Hypothesen stützen oder widerlegen. Eine Verbindung zu anderen Studienergebnissen wird hergestellt.

\section{Unterschiede zwischen FC-Anwendende und Nicht-Anwendende}

\section{Selbstwirksamkeit:}

Die Teilnehmenden der Stichprobe, die in ihrer Praxis bereits mit FC arbeiten, berichten ein stärkeres Gefühl der Selbstwirksamkeit als jene, die FC noch nicht in der Unterrichtspraxis nutzen. In Anlehnung an Van Acker et al. (2013) wurde für diesen Beitrag unter Selbstwirksamkeit das Wissen über OER, der Nutzen der Materialien für andere sowie die notwendigen technischen Fähigkeiten zur Produktion und weiteren Verbreitung von Materialien summiert. Die Selbstwirksamkeit ist ein entscheidender Prädiktor, ob Lehrpersonen überhaupt Materialien erstellen und teilen (vgl. Van Acker et al. 2013, 188).

\section{Bereitschaft zu teilen:}

Obwohl sich die FC-Praktizierenden in der Stichprobe bezüglich der Selbstwirksamkeit höher einschätzen, unterscheiden sie sich nicht signifikant von der Nicht-FCGruppe hinsichtlich des Items Bereitschaft zu teilen. Auf den ersten Blick scheint dies den Ergebnissen von Van Acker et al. (2013), Selbstwirksamkeit wird als entscheidender Faktor für das Teilen von OER genannt, zu widersprechen. Betrachtet man die gefundenen Ergebnisse im Detail, wird jedoch ersichtlich, dass die Standardabweichung bei der Nicht-FC-Gruppe mit 1.0 relativ hoch ist. Dies bedeutet, dass es auch in der Nicht-FC-Gruppe durchaus Personen geben muss, die über viel Wissen zu OER und über die entsprechenden Fähigkeiten zur Erstellung und Verbreitung von Materialien verfügen.

Offenheit:

Um auch etwas über die Qualität der Teil-Bereitschaft aussagen zu können, wurde die Stichprobe befragt, mit welcher Lizenz produzierte Materialien gekennzeichnet 
werden. Hier verringert sich die Anzahl der vollständig ausgefüllten Fragebögen in beiden Gruppen, da bei (noch) keinem Wissen über die Lizenzmodelle oder fehlender Antwortbereitschaft oder -möglichkeit (eine Entscheidung hinsichtlich der Lizenzierung wird von Fall zu Fall neu entschieden) die Antworten nicht berücksichtigt werden. Für die FC-Gruppe verbleiben damit 118 ausgefüllte Fragebögen, für die NichtFC-Gruppe 58. Bezüglich der Lizenzierung ist ein signifikanter Unterschied zwischen FC- und Nicht-FC-Gruppe erkennbar. Auch ein Zusammenhang von Selbstwirksamkeit und der Lizenzierung liegt vor ( $r s=0.396, p=0.000$ ).

Die vorliegenden Ergebnisse unterscheiden sich zu denen von de los Arcos (2014). Hier gaben lediglich knapp 5\% der befragten FC-Anwendenden an, CC-Lizenzen bei der Weitergabe der erstellten Materialien zu vergeben. In der vorliegenden Erhebung tun dies knapp über $80 \%$ (58\% CC BY-SA, 25\% CC BY-ND) und fünf Prozent nutzen sogar CC0, verzichten also auch auf die Namensnennung (iRights.info o.J.). Einschränkend muss hier festgehalten werden, dass diese Daten noch nicht berücksichtigen, ob die Materialien tatsächlich selbst produziert und dann weitergegeben werden. Dieser Umstand wird in der Diskussion der zweiten Hypothese behandelt.

Zusammengefasst kann festgehalten werden, dass die in der Erhebung gefundenen Werte Hypothese 1 teilweise stützen können. Es wurde kein signifikanter Unterschied hinsichtlich der Bereitschaft zu teilen zwischen FC-Anwendenden und Nicht-Anwendenden gefunden. FC-Anwendende schätzen ihr Wissen zu OER sowie ihre technischen Fähigkeiten zur Erstellung und zum Teilen von OER-Materialien höher ein; diese Einschätzung hängt wiederum mit der Qualität der Lizenzierung zusammen. So nutzen FC-Anwendende signifikant häufiger Lizenzen, die sowohl die Bearbeitung als auch Weitergabe von Materialien erlauben. In der Stichprobe kann dies mit dem Wissen über die vorhandenen Lizenzmodelle erklärt werden.

\section{Unterschiede zwischen den FC-Anwenderinnen und -Anwendern}

\section{Selbstwirksamkeit:}

In der Stichprobe geben 120 FC-Anwenderinnen und -Anwender an, selbst Materialien für die Umsetzung von FC zu produzieren, 34 produzieren nicht selbst. Die beiden Gruppen unterscheiden sich signifikant hinsichtlich ihrer eingeschätzten Selbstwirksamkeit. Die FC-Praktizierenden verfügen demnach über ein höheres Wissen zu OER, besitzen bessere technische Fähigkeiten zur Produktion und zum Teilen und glauben stärker daran, dass ihre Materialien anderen Lehrpersonen im Unterricht oder der Vorbereitung helfen können. Dies deckt sich mit den Ergebnissen bei Van Acker et al. (2013). Das Gefühl, OER könnten anderen helfen, spielt für das Teilen eine wichtige Rolle, und ist wichtiger als das Gefühl, dass Lehrpersonen damit ihr Renommee verbessern können (vgl. Van Acker et al. 2013, 188). 
Bereitschaft zu teilen:

Signifikante Unterschiede zeigen sich in der Stichprobe für die Bereitschaft zu teilen innerhalb der FC-Gruppe. Werden für FC eigene Materialien produziert, steigt demnach die Bereitschaft, diese auch zu teilen. Bei de los Arcos (2014) gaben 43,3\% der Lehrpersonen der Stichprobe an, die selbst erstellten Materialien auch online zu publizieren und zu teilen. In der vorliegenden Erhebung im deutschsprachigen Raum sind es 62,2\%, die die Werte 1 oder 2 auf der Likert-Skala ( 1 = stimme ganz zu) innerhalb der Skala Bereitschaft zu teilen gewählt haben.

\section{Offenheit:}

Bei der Vergabe der Lizenzen unterscheiden sich die FC-Praktizierenden mit Eigenproduktion erneut signifikant von den FC-Anwendenden, die keine eigenen Materialien produzieren. Hier können die Ergebnisse dieser Erhebung einem direkten Vergleich mit jenen von de los Arcos (2014) unterzogen werden. Gaben bei ihr lediglich $5,1 \%$ der produzierenden FC-Anwendenden an, die selbst erstellten Materialien mit einer CC-Lizenz zu kennzeichnen, tun dies in dieser Stichprobe 86\% (66\% CC BY-SA, $20 \%$ CC BY-ND), 7\% verwenden CC0. Auffällig ist, dass die nicht-produzierenden FCAnwendenden fünf Mal häufiger eigens produzierte Materialien mit $\odot$ kennzeichnen würden als in der FC-Gruppe, die selber produziert.

Die gefundenen Befunde bestätigen Hypothese 2: FC-Anwendende, die Materialien selbst produzieren, unterscheiden sich in allen drei skizzierten Bereichen von jenen Anwendenden, die keine eigenen Materialien herstellen.

Wird die Gruppe der Nicht-FC-Anwendenden als Kontrollgruppe herangezogen, finden sich signifikante Unterschiede lediglich für die FC-Gruppe mit Produktion. Die FC-Gruppe ohne eigene Produktion unterscheidet sich in keinem Bereich signifikant von jenen Personen, die FC nicht praktizieren.

Alleine der Einsatz von FC in der Praxis kann auf der Basis der hier gefundenen Ergebnisse nicht als Prädiktor für die Bereitschaft zu teilen angesehen werden. Auch sorgt die blosse Anwendung von FC nicht dafür, dass Materialien anderen unter CCLizenzen zur Verfügung gestellt werden.

FC-Anwendende scheinen sich jedoch intensiver mit der Thematik OER im Allgemeinen auseinanderzusetzen. Die in dieser Stichprobe abgegebenen Antworten von FC-Praktizierenden deuten auf jeden Fall darauf hin, berichten sie doch über mehr Wissen zu OER und besser ausgebildete technische Fähigkeiten und schätzen die Hilfe, die sie durch das Teilen mit anderen leisten können, höher ein. Hervorzuheben ist die Gruppe der FC-Anwendenden, die eigene Materialien produziert. Sie unterscheidet sich sowohl von den Nicht-Anwendenden als auch den FC-Anwendenden ohne Produktion sowohl hinsichtlich der Selbstwirksamkeit, der Bereitschaft zu teilen als auch der Offenheit signifikant. 
Ein Vergleich zwischen FC-Anwendenden mit Produktion und Lehrpersonen mit gut ausgebildeten technischen Fähigkeiten oder solchen, die Materialien für andere Unterrichtskonzepte, Kontexte, etc. produzieren, fehlt. Daher kann nicht ausgeschlossen werden, dass die Produktion alleine zur erhöhten Bereitschaft zu teilen und der Verwendung offener(er) Lizenzen führt.

\section{Zusammenfassung und Ausblick}

Die vorgestellte Studie untersucht die Nutzung und Erstellung von Open Educational Resources im konkreten methodisch-didaktischen Settings des Flipped Classroom und unternimmt damit den Versuch, ein von Deimann (2018) implizit aufgezeigtes Forschungsdesiderat zu schliessen. Dabei schliesst sie an die von Ebner et al. (2015) durchgeführte Ist-Analyse zu freien Bildungsmaterialien (OER). Die Situation von freien Bildungsmaterialien (OER) in Deutschland in den Bildungsbereichen Schule, Hochschule, berufliche Bildung und Weiterbildung im Juni 2015 sowie die von de los Arcos (2014) im US-amerikanischen Raum durchgeführte Studien an.

Die beiden der Untersuchung zugrunde gelegten Forschungshypothesen sind unter Aufnahme der von de los Arcos (2014) für den US-amerikanischen Raum gefundenen Ergebnisse formuliert, fokussieren jedoch den deutschsprachigen Raum:

Hypothese 1: Lehrende, die das Modell des Flipped Classroom (FC) praktizieren, unterscheiden sich von Nicht-Praktizierenden in den Bereichen:

a. Selbstwirksamkeit (bezogen auf Wissen und Fähigkeiten zu OER)

b. Bereitschaft zu teilen

c. Offenheit (bezogen auf die Auswahl der Lizenz)

Hypothese 2: Lehrende, die Materialien für den FC selbst erstellen, unterscheiden sich in den drei oben genannten Aspekten von jenen FCAnwender*innen, die ihre Materialien nicht selber erstellen.

Zunächst wurde hierfür erhoben, wer überhaupt den umgedrehten Unterricht in deutschsprachigen Bildungseinrichtungen anwendet. Das untersuchte Sample ( $N=$ 361) teilt sich in 191 FC-Anwendende und 170 Nicht-FC-Anwendende. Es zeigt sich, dass besonders im tertiären Bildungsbereich nach diesem Konzept gelehrt wird, aber auch in der Sekundarstufe 2 eine grosse Anzahl an Lehrkräften mittlerweile mit Flipped Classroom unterrichtet.

Was Hypothese 1 betrifft, so zeigt sich, dass die Gruppe der FC-Anwendenden eigenen Angaben zufolge in Hinblick auf OER und FC viel Wissen sowie gut ausgebildete technische Fähigkeiten besitzt und bereit ist, Materialien unter Lizenzen weiterzugeben, die anderen die Wiederverwendung und (meist) auch Bearbeitung 
ermöglicht. Im Bereich Selbstwirksamkeit und Offenheit konnten zwischen den beiden Gruppen signifikante Unterschiede erkannt werden, in der Bereitschaft zu teilen sind die Unterschiede nicht signifikant.

Für Hypothese 2 wurde das Sample auf jene FC-Anwendende eingeschränkt ( $n=$ 154), die eigene Materialien erstellen $(n=120)$ und jene, die Materialien nicht selbst erstellen $(n=34)$. Hier wurden signifikante Unterschiede in allen drei Teilbereichen erkannt. Die vorliegende Erhebung weist hier Unterschiede zur Erhebung von de los Arcos (2014) auf. So kann festgehalten werden, dass FC-Anwendende im deutschsprachigen Raum allgemein eher dazu bereit sind, offene(re) Lizenzen für geteilte Materialien zu vergeben. Wenn diese FC-Anwendende eigene Materialien produzieren, zeigen sie eine höhere Bereitschaft zu teilen als Nicht-FC-Anwendende und FCAnwendende ohne eigene Produktion; sie tendieren dazu, CCO, CC BY-SA oder CC BYND zur Lizenzierung ihrer erstellten Materialien zu verwenden.

Die Ergebnisse dieser quantitativen Erhebung lassen keine Rückschlüsse auf die Gründe zu, warum Materialien geteilt werden und welche Motive hinter einem Teilen als OER oder nicht stehen. Eine an die Ergebnisse dieser Erhebung anschliessende qualitative Studie wäre wünschenswert, die die Argumentation von Van Acker et al. (2013) überprüft, die in ihrer Studie berichten, dass extrinsische Anreize für Lehrpersonen nicht entscheidend bei der Bereitschaft zu teilen sind. Vielmehr zeichnen sich solche Lehrpersonen durch intrinsische Motiviertheit aus (vgl. Van Acker et al. 2013, 187).

\section{Limitationen}

Die vorliegende Studie kann als erster Einblick in die Thematik gesehen werden und weist einige Einschränkungen auf, die in Folgestudien zu beachten wären: Eine zentrale Limitation der vorliegenden Studie ist in der Stichprobengrösse zu sehen. Gerade beim Vergleich innerhalb der FC-Gruppe gibt es grosse Unterschiede bei der Anzahl der berücksichtigten Fragebögen, besonders beim Vergleich zum Item Offenheit.

Auch das Vorgehen bei der Auswahl der Stichprobe muss kritisch hinterfragt werden. So wurde über die zur Distribuierung gewählten (und zur Verfügung gestandenen) Kanäle nicht die «Durchschnittslehrperson» erreicht. Auf Twitter, im Newsletter der Virtuellen Pädagogischen Hochschule und über die E-Mail-Liste von eEducation Austria werden vorrangig jene Lehrpersonen erreicht, die sich bereits mit Themen wie $O E R, F C$, Bildung in der digitalen Welt u.ä. beschäftigen. Aussagen für die Allgemeinheit der Lehrpersonen in der Schule können ebenso wenig getroffen werden, wie für Lehrende an Hochschulen, da gerade eEducation Austria, aber auch die Virtuelle Pädagogische Hochschule von der Zielgruppe her schwerpunktmässig in der Sekundarstufe anzusiedeln sind. 
Wie Van Acker et al. (2013) zeigen, spielen bei der Bereitschaft zu teilen mehrere Faktoren zusammen: Die von Van Acker et al. (2013) genannten möglichen Kosten oder Belohnungssysteme, z.B. Beförderungen oder monetäre, wurden in dieser Erhebung ebenso wenig berücksichtigt wie das Vertrauen darin, dass andere Lehrende ebenfalls teilen, wenn ich es tue.

\section{Desiderate}

Aus der vorliegenden Untersuchung ergeben sich Forschungsdesiderate, die zum einen aus den Limitationen abgeleitet werden können, wie eine umfassende Untersuchung, deren Datenbeschaffung nicht über digitale Kanäle und Social Media erfolgt, und eine qualitative Untersuchung, die sich mit der inneren Wertehaltung und Einstellung der Befragten beschäftigt.

Ein weiteres Forschungsdesiderat leitet sich aus dem weiten Verständnis des Begriffs Offenheit ab. Wie Jöran Muuß-Merholz und Sandra Schön $(2015,39)$ gezeigt haben, ist die subjektive Wahrnehmung von Offenheit oftmals mit freier Zugänglichkeit im Internet gleichzusetzen. Ein derartiges Verständnis ist auch innerhalb der dieser Arbeit zugrundeliegenden Untersuchung zu vermuten, die Item-Formulierung im Fragebogen war jedoch zu vage, als dahingehend interpretierbare Antworten ableitbar wären. Eine qualitative Untersuchung würde hier weiteren Aufschluss geben, der im Bereich der OER-Forschung eine zentrale Bedeutung haben könnte, insofern als Schulungs- und Informationsbedarfe identifiziert werden könnten.

Ein weiteres Forschungsdesiderat schliesst an die Untersuchung von Neumann (2016) an, die vermuten lässt, dass gerade im Bereich der Fremdsprachen viel über offene Bildungsressourcen gesprochen wird. Die in Tabelle 1 gegebene Übersicht zeigt jedoch, dass gerade das Mathematisch-Analytische FC-affin zu sein scheint. Eine genauere Betrachtung der unterschiedlichen Fächer sowohl in Hinblick auf den Einsatz des FC-Modell als auch die Verwendung und Erstellung von OER wäre interessant und wünschenswert. Neigen Fremdsprachenlehrende zur Erstellung und Nutzung von OER, nicht aber zur Nutzung des FC? Und ist die Situation bei Mathematiklehrenden umgekehrt? Oder wird im Sprachenunterricht FC betrieben, ohne inn als solchen zu bezeichnen? Ergebnisse, die sich aus der Beantwortung dieser Fragen ergeben, könnten einen wertvollen Beitrag für die Lehrpersonenausbildung, die sich in Österreich gerade im Umbruch befindet, leisten.

Das (offene) Teilen von Materialien zur Ermöglichung der Individualisierung und Binnendifferenzierung in einer schnelllebigen Wissensgesellschaft wäre erstrebenswert, hierfür wäre die Erhebung von Anreizmöglichkeiten wünschenswert. Ziel wäre, Lehrpersonen stärker zu vernetzen und ein Bewusstsein und eine Kultur des «Sharing», über die Gruppe der FC-Anwendenden hinaus, zu etablieren. Sowohl innerhalb der Grenzen der eigenen Schule als auch im Internet und den dort zur Verfügung 
stehenden Vernetzungsplattformen. Erfahrene Flipped-Classroom-Anwendernde, die sich in Offenheit und Selbstwirksamkeit signifikant von Nicht-Anwendenden unterscheiden, könnten interessierte Kolleginnen und Kollegen durch das (offene) Zur-Verfügung-Stellen ihrer Materialien unterstützen und die Hürden für ein erstmaliges Ausprobieren, sowohl im Einsatz des FC als auch im Erstellen von OER, verringern. Die vorliegende Untersuchung gibt erste Aufschlüsse darüber, dass FC und OER Parameter sind, die in einem Naheverhältnis zueinanderstehen können. Die FCAnwendenden sind durch die Adaption und Produktion von Materialien, die auf ihre Lerngruppen zugeschnitten sind (v.a. auch im Bereich Video-Content) für das Thema $O E R$ sensibilisiert. Sie könnten für Nicht-OER-Erstellerinnen und -Ersteller und NichtFC-Anwendende gleichsam als Vorreiterinnen und Vorreiter fungieren, hierfür sollten jedoch individuelle Motivationen und Wertehaltungen erhoben werden, um auch Bedarfe in Hinblick auf die Entwicklung von Schulungen und Anreizsystemen oder Distributionskanälen zu kennen.

\section{Literatur}

Bergmann, Jonathan, und Aaron Sams. 2012. Flip Your Classroom: Reach Every Student in Every Class Every Day. Washington, DC: International Society for Technology in Education.

Buchner, Josef, und Christian Freisleben-Teutscher. 2017. «Offene Bildungsressourcen im/mit dem Inverted Classroom Modell». In Inverted Classroom - The Next Stage: Lehren und Lernen im 21. Jahrhundert, herausgegeben von Sabrina Zeaiter und Jürgen Handke, 115-22. Baden-Baden: Tectum.

De los Arcos, Beatriz. 2014. «Flipped Learning and OER: Survey Results». open education et cetera (blog). 2014. https://oscailte.wordpress.com/2014/03/13/research-findings-on-flippedlearning-and-oer/.

De los Arcos, Beatriz, Robert Farrow, Rebecca Pitt, Martin Weller, und Patrick Mcandrew. 2016. «Personalising Learning through Adaptation: Evidence from a Global Survey of K-12 Teachers' Perceptions of Their Use of Open Educational Resources.» Journal of Online Learning Research 2 (1): 23-40.

Deimann, Markus. 2018. «OER-Forschung - Warum es sie bisher nicht gab und wie sich das ändern kann». Synergie, Nr. 5: 71-75. https://uhh.de/xk0cw.

Ebner, Martin, Elly Köpf, Jöran Muuß-Merholz, Martin Schön, Sandra Schön, und Nils Weichert. 2015. IST-Analyse zu freien Bildungsmaterialien (OER). Die Situation von freien Bildungsmaterialien (OER) in Deutschland in den Bildungsbereichen Schule, Hochschule, berufliche Bildung und Weiterbildung im Juni 2015. Books on Demand.

Fischer, M., und Christian Spannagel. 2012. «Lernen mit Vorlesungsvideos in der umgedrehten Mathematikvorlesung». In DeLFI 2012 - Die 10. e-Learning Fachtagung Informatik der Gesellschaft für Informatik e. V., 225-36. Bonn: Köllen Druck+Verlag. http://www01.ph-heidelberg. de/wp/spannagel/publications/Fischer_Spannagel_2012.pdf. 
Geser, Guntram. 2007. «Open Educational Practices and Resources». Salzburg Research. http:// www.salzburgresearch.at/research/publications_detail.php?pub_id=357.

Goodier, Sarah. 2017. «Tracking the Money for Open Educational Resources in South African Basic Education: What We Don't Know». The International Review of Research in Open and Distributed Learning 18 (4). https://doi.org/10.19173/irrodl.v18i4.2990.

Handke, Jürgen. 2014. Patient Hochschullehre. Vorschläge für eine zeitgemäße Lehre im 21. Jahrhundert. Marburg: Tectum Verlag.

Hankmann, Marc. 2014. «Frei, kostenlos - aber auf Halde. Potenzial von OER und warum sie so selten für den Unterricht genutzt werden.» Bildung + Bildung spezial 4 (4).

Hassler, Bjoern, Sara Hennessy, und Riikka Hofmann. 2018. «Sustaining and Scaling Up Pedagogic Innovation in Sub-Saharan Africa: Grounded Insights For Teacher Professional Development». Journal of Learning for Development 5 (1): 58-78. https://jl4d.org/index.php/ejl4d/ article/view/264.

Heimstädt, Maximilian, und Leonhard Dobusch. 2017. «Perspektiven von Open Educational Resources (OER) für die (sozio-)ökonomische Bildung an Schulen in NRW und in Deutschland». 2017. http://www.fgw-nrw.de/forschungsergebnisse/forschungsergebnisse/projektdetails/ news/perspektiven-von-open-educational-resources-oer-fuer-die-sozio-oekonomischebildung-an-schulen-in.html.

iRights.info. 2019. «Was ist Creative Commons Zero?» 7. Dezember 2019. https://irights.info/ artikel/was-ist-cc0/28750.

Kerres, Michael. 2016. «Open Educational Resources». In Enzyklopädie der Wirtschaftsinformatik, 9. Auflage. Berlin. https://www.enzyklopaedie-der-wirtschaftsinformatik.de/lexikon.

Kimmons, Royce. 2015. "OER Quality and Adaptation in K-12: Comparing Teacher Evaluations of Copyright-Restricted, Open, and Open/Adapted Textbooks.»International Review of Research in Open and Distributed Learning 16 (5): 39-57. https://doi.org/10.19173/irrodl. v16i5.2341.

Lage, Maureen J., Glenn J. Platt, und Michael Treglia. 2000. «Inverting the Classroom: A Gateway to Creating an Inclusive Learning Environment». The Journal of Economic Education 31 (1): 30-43. https://doi.org/10.2307/1183338.

Lo, Chung Kwan, Khe Foon Hew, und Gaowei Chen. 2017. «Toward a Set of Design Principles for Mathematics Flipped Classrooms: A Synthesis of Research in Mathematics Education». Educational Research Review 22 (November): 50-73. https://doi.org/10.1016/j.edurev.2017.08.002.

Longhino, Daniela. 2018. Offener Unterricht: Qualitätsmerkmale, Dimensionen und Unterrichtskonzept. https://www.schule-im-aufbruch.at/wp-content/uploads/Schule\%20im\%20Aufbruch_Publikation\%201_Longhino_04\%2001\%2018.pdf.

Mardis, Marcia A, und Chandrahasa R Ambavarapu. 2017. «Usage Data as Indicators of OER Utility». Journal of Online Learning Research 3 (2): 197-221. 
Muuß-Merholz, Jöran, und Sandra Schön. 2015. «Freie Bildungsmaterialien (OER) in der Schule». In IST-Analyse zu freien Bildungsmaterialien (OER). Die Situation von freien Bildungsmaterialien (OER) in Deutschland in den Bildungsbereichen Schule, Hochschule, berufliche Bildung und Weiterbildung im Juni 2015., herausgegeben von Martin Ebner, Elly Köpf, Jöran Muuß-Merholz, Martin Schön, Sandra Schön, und Nils Weichert, 35-76. Books on Demand.

Neumann, Dominik. 2016. Open Educational Resources (OER) oder Kostenloses Lehrmaterial aus dem Internet. Marktanalyse 2016 und Aktualisierung der Diskussion. https://www.pedocs.de/ volltexte/2016/12671/pdf/Neumann_2016_OER.pdf.

O'Flaherty, Jacqueline, und Craig Phillips. 2015. «The use of flipped classrooms in higher education: A scoping review». Internet and Higher Education 25: 85-95. https://doi.org/10.1016/j. iheduc.2015.02.002.

Peschel, Falko. 2012. Offener Unterricht. Teil I+II. Schneider Verlag Hohengehren.

Peter, Sandra, und Markus Deimann. 2013. «On the Role of Openness in Education: A Historical Reconstruction». Open Praxis 5 (1): 7-14. https://doi.org/10.5944/openpraxis.5.1.23.

Schön, Sandra. 2018. «Replik auf Deimann (2018) - natürlich gibt es Forschung zu OER». 2018. https://sansch.wordpress.com/2018/06/11/replik-auf-deimann-2018-natuerlich-gibt-esforschung-zu-oer/.

Schön, Sandra, Katharina Kreissl, Leonhard Dobusch, und Martin Ebner. 2017. Mögliche Wege zum Schulbuch als Open Educational Resources (OER). Eine Machbarkeitsstudie zu OERSchulbüchern in Österreich. Salzburg. https://www.salzburgresearch.at/wp-content/uploads/2017/11/machbarkeitsstudie_submission_final.compressed.pdf.

Treeck, Timo van, Klaus Himpsl-Gutermann, und Jochen Robes. 2013. «Offene und partizipative Lernkonzepte: E-Portfolios, MOOC's und Flipped Classrooms». In Lehrbuch für Lernen und Lehren mit Technologien, herausgegeben von Martin Ebner und Sandra Schön. http://l3t.eu.

Van Acker, Frederik, Hans Van Buuren, Karel Kreijns, und Marjan Vermeulen. 2013. "Why teachers share educational resources: A social exchange perspective». In Perspectives on open and distance learning, herausgegeben von Rory McGreal, Wanjira Kinuta, und Stewart Marshall, 177-92. Commonwealth of Learning and Athabasca University.

Van Acker, Frederik, Marjan Vermeulen, Karel Kreijns, Jan Lutgerink, und Hans van Buuren. 2014. «The role of knowledge sharing self-efficacy in sharing Open Educational Resources». Computers in Human Behavior 39: 136-144. https://doi.org/10.1016/j.chb.2014.07.006.

Wampfler, Philippe. 2015. «Warum teilen Lehrkräfte ihre Materialien nicht?» Schule Social Media. 2015. https://schulesocialmedia.com/2015/03/29/warum-teilen-lehrkrafte-ihre-materialien-nicht/.

Weller, Martin, Beatriz De los Arcos, Rob Farrow, Beck Pitt, und Patrick McAndrew. 2015. «The Impact of OER on Teaching and Learning Practice». Open Praxis 7 (4). https://doi.org/10.5944/ openpraxis.7.4.227.

Wiley, David. 2014. «The Access Compromise and the 5th R.» 2014. https://opencontent.org/ blog/archives/3221.

Zöhrer, Helmut. 2016. «Einsatz von Open Educational Resources mittels Flipped Classroom im Mathematikunterricht». Diplomarbeit. 